\section{BMJ Open Respiratory Research}

\title{
Two chair test: a substitute of 6 min walk test appear cardiopulmonary reserve specific
}

Parthasarathi Bhattacharyya (D) , ${ }^{1}$ Dipanjan Saha, ${ }^{2}$ Mintu Paul, ${ }^{2}$ Dhiman Ganguly, ${ }^{3}$ Biswarup Mukherjee, ${ }^{4}$ Sushmita Roy Chowdhury, ${ }^{5}$ Sourav RoyChoudhury, ${ }^{6}$ Pawan Agarwal, ${ }^{5}$ Indranil Halder, ${ }^{7}$ Debanu Ghosh Roy, ${ }^{8}$ Shuvanan Ray ${ }^{9}$

\section{ABSTRACT}

To cite: Bhattacharyya $P$, Saha D, Paul M, et al. Two chair test: a substitute of 6 min walk test appear cardiopulmonary reserve specific. BMJ Open Resp Res 2020;7:e000447. doi:10.1136/ bmjresp-2019-000447

Received 8 June 2019 Revised 13 December 2019 Accepted 31 January 2020
Check for updates

(c) Author(s) (or their employer(s)) 2020. Re-use permitted under CC BY-NC. No commercial re-use. See rights and permissions. Published by BMJ

For numbered affiliations see end of article.

Correspondence to Dr Parthasarathi Bhattacharyya; parthachest@yahoo.com
Background A simple and efficient exercise test possible in a small space is welcome to supplement 6 min walk test (6MWT) that demands a 100 feet corridor to perform. Methods The proposed two chair test (2CT) makes a person to sit and move five times between two chairs placed face to face at 5 feet apart and note the changes in pulse-rate (PR) and arterial oxygen saturation $\left(\mathrm{SpO}_{2}\right)$ at every $10 \mathrm{~s}$ for $2 \mathrm{~min}$ after that. Comparison of the post-exercise measurements ( $\mathrm{PR}$ and $\mathrm{SpO}_{2}$ ) with a repeat performance in same patients was done for reproducibility and doing the same after 6MWT and 2CT in another set of patients was meant for for acceptability. The statistical analysis was made on moment to moment change, mean maximal difference and mean cumulative difference for the measurements using $p$ value, $z$-score, $r$ value and principal component analysis (PCA).

Findings $A$ total of 40 and 60 volunteers were included for testing reproducibility and acceptability. On both the sets, the difference in most of comparisons between the measured variable ( $\mathrm{PR}$ and $\mathrm{SpO}_{2}$ ) showed the $\mathrm{p}$ values remaining insignificant $(>0.05)$, and z-score being $<1$ SD of the corresponding other and the correlation coefficients (r) remaining excellent $(>0.9)$. Furthermore, the PCA shows complete overlapping. The post-exercise changes did not corelate the walking distance in 6MWT.

Interpretation The proposed 2CT demands small space and appears reproducible and comparable with 6MWT in terms of its post-exercise impact on PR and $\mathrm{SpO}_{2}$. This novel test also appears more of cardiopulmonary reserve specific.

\section{INTRODUCTION}

Six minutes walk test (6MWT) is a submaximal exercise test has been popular in the domain of cardio-respiratory diseases. ${ }^{1-16}$ It has been found helpful in pretreatment and post-treatment comparison in pulmonary rehabilitation, ${ }^{12}$ heart failure, ${ }^{3}$ chronic obstructive pulmonary disease (COPD), ${ }^{4}$ pulmonary hypertension, ${ }^{5}$ lung surgery ${ }^{67}$ and lung volume reduction. ${ }^{8}$ Even a single measurement of the test has been proved useful to understand the functional status of patients with several conditions, such as COPD, ${ }^{9}$

\section{Key messages}

Can we make a simple and reproducible exercise test that can substitute 6 min walk test (6MWT)?

- Two chair test can potentially substitute 6MWT.

- It is simple, reproducible, comparable to 6MWT as regards the post-exercise recovery response in terms of pulse rate and saturation, and performable in a consultation room itself.

cystic fibrosis, ${ }^{10}$ heart failure ${ }^{11}$ and peripheral vascular disease, ${ }^{12}$ Finally, and most importantly, the test has been proved as a predictor of mortality in heart failure, ${ }^{13} \mathrm{COPD}^{1415}$ and primary pulmonary hypertension. ${ }^{16}$

The distance covered in the process is noted as $6 \mathrm{~min}$ walking distances (6MWD) that reflects global physiological response to exercise. Hence, it is not a cardio-respiratory specific exercise test to appreciate the ability or debility of a subject with symptoms of cardiopulmonary compromise. Moreover, the test demands an unobstructed indoor walking space of over 100 feet. ${ }^{17}$ This remains a logistic constraint in many situations. Hence, there is an unmet need of an easily performable but efficient substitute of 6MWT that require minimum space and less time to perform. There were efforts to evolve such substitutes as 3 min step test, ${ }^{18}$ shuttle walk test, ${ }^{19}{ }^{20}$ sit to stand test but none could match the acceptance of $6 \mathrm{MWT}^{21}$ This manuscript deals with another novel effort, the 2 chair test (2CT), meant to substitute the 6MWT.

\section{METHODS}

\section{Decision for and description of the test (2CT)}

The prospective test was discussed in details at the planning stage itself. A team of pulmonologists and cardiologists (Kolkata $\mathrm{PH}$ group) finally decided in favour of 2CT (see later) on consensus after considering other feasible 
options been proposed. A pilot survey on its performance was also reviewed by the group. The group decided to establish the reproducibility of 2CT and also to compare it with 6MWT before finally accepting it. It was decided to have two sets of patients in this pilot observation (each having a numerical strength $>30$ ) for testing reproducibility and for comparison with 6MWT on available physiological parameters in a common and defined fashion to compare. A good comparability was taken as 'acceptability' of the test. The decision process took into consideration the space requirement, the ease of performing the test and the concerned safety issues.

The proposed test was termed as 'two chair test' (2CT). It involved a person to sit and walk between two chairs as an exercise and the physiological impact is quantitatively measured in terms of change in arterial oxygen saturation $\left(\mathrm{SpO}_{2}\right)$ and pulse-rate (PR) counted post-exercise. Two ordinary wooden chairs (with flat back-rest, no hand rest, the sitting surface being $48 \mathrm{~cm}$ above the ground level) are to be placed face to face at a distance of 5 feet (from anterior to anterior ends) facing each other. First, a patient needs to sit on any one of them and rest for a few minutes till his $\mathrm{PR}$ and $\mathrm{SpO}_{2}$ remains stable continuously at least for $30 \mathrm{~s}$. At this point, the baseline PR and the $\mathrm{SpO}_{2}$ are recorded and the patient is then asked to get up and walk to the other chair and sit there before getting up and returning to the previous chair. This is recorded as single movement. The patient is directed to make five similar movements otherwise continuously at his/her own pace with freedom to stop in between for dyspnoea or any physical discomfort or fatigue. There is a provision for premature termination of the test following the recommendation by American Thoracic Society on 6MWT for chest pain, intolerable dyspnoea, leg cramps, staggering, turning pale or ashen, and diaphoresis. ${ }^{17}$ In case of stopping in between or premature termination, it is mandatory to note the number of movements and details of events including the duration, reasons, status as $\mathrm{PR}$, blood pressure and $\mathrm{SpO}_{2}$. The contraindications for the test were kept as the same for $6 \mathrm{MWT} .{ }^{17}$ The patients were allowed to use their anti-angina or other drugs as routine without any modification of the schedule and emergency use of nitro-glycerine was allowed, if necessary. Oxygen supplementation was offered to those who required prolonged time (more than $30 \mathrm{~s}$ ) to show signs of recovery from desaturation or had severe desaturation $\left(\mathrm{SpO}_{2}<80 \%\right)$. After completing the five movements $\mathrm{PR}$ and $\mathrm{SpO}_{2}$ were measured and recorded immediately and thereafter at an interval of every $10 \mathrm{~s}$ for next $2 \mathrm{~min}$. The discussed commonalities and differences of 2CT with $6 \mathrm{MWT}$ as perceived by the group was detailed (see table 1).

The decision for five movements were made on the basis of a pilot observation on six volunteers (patients) who were subjected to 4, 5 and 6 movements in 2CT and also the 6MWT with adequate rest $(30 \mathrm{~min})$ in between the performances. The cumulative changes in PR and $\mathrm{SpO}_{2}$ after five movements showed best corelationship

\begin{tabular}{|c|c|c|}
\hline Qualities & 6MWT & $2 \mathrm{CT}$ \\
\hline Physiological measurement & Walking distance & Post-exercise change in PR and $\mathrm{SpO}_{2}$ \\
\hline Assessment & Functional capacity & $\begin{array}{l}\text { Cardiopulmonary response and } \\
\text { decompensation }\end{array}$ \\
\hline Nature & $\begin{array}{l}\text { Sub-maximal exercise; walking in } \\
100 \text { feet corridor }\end{array}$ & $\begin{array}{l}\text { Sub-maximal exercise; changing seating from } \\
\text { one chair to other five times }\end{array}$ \\
\hline Pace & Self-controlled & Self-controlled \\
\hline Duration of exercise & $6 \min$ & No fixed duration \\
\hline Pre-performance direction & Given & Given \\
\hline Premature termination & Allowed & Allowed \\
\hline Encouragement during performance & Not allowed & Not allowed \\
\hline $\begin{array}{l}\text { Oxygen supplementation, if necessary, } \\
\text { after or at premature termination }\end{array}$ & Allowed & Allowed \\
\hline Looked for & Distance covered & $\begin{array}{l}\text { Post-exercise desaturation and change in PR } \\
\text { (pulse rate) for } 2 \mathrm{~min}\end{array}$ \\
\hline Space required & Long walking space (hall/corridor) & Consultation room \\
\hline Other instruments & $\begin{array}{l}\text { BP machine, and a } \\
\text { pulse oximeter }\end{array}$ & $\begin{array}{l}\text { Two chairs, and a } \\
\text { pulse oximeter }\end{array}$ \\
\hline Emergency back up & Oxygen and emergency medicines & Oxygen and emergency medicines \\
\hline Personnel & Trained for basic life support & Trained for basic life support \\
\hline Safety & Safe & Likely safe \\
\hline
\end{tabular}

Elaborates the basic commonalities and differences between 2CT and 6MWT

$\mathrm{BP}$, blood pressure; $2 \mathrm{CT}$, two chair test; $6 \mathrm{MWT}, 6$ min walk test; $\mathrm{PR}$, pulse-rate; $\mathrm{SpO}_{2}$, arterial oxygen saturation. 
Table 2 The comparison between 4, 5 and 6 movements in two chair test (2CT) with 6MWT in terms of cumulative changes in pulse-rate and $\mathrm{SpO}_{2}$

\begin{tabular}{|c|c|c|c|c|}
\hline \multirow[b]{2}{*}{$n=6$} & \multicolumn{4}{|c|}{ Number of movements } \\
\hline & After 6MWT & 4 & 5 & 6 \\
\hline Mean cumulative pulse change & $55.14 \pm 46.64$ & $43.07 \pm 45.84$ & $63.64 \pm 55.71$ & $74.5 \pm 61.82$ \\
\hline Mean cumulative SPO2 change per patient & $-6.5 \pm 4.25$ & $-4.92 \pm 2.67$ & $-8.28 \pm 4.77$ & $-8.35 \pm 6.33$ \\
\hline Cumulative pulse change & 772 & 603 & 891 & 1043 \\
\hline Cumulative $\mathrm{SpO}_{2}$ change & -91 & -69 & -116 & -117 \\
\hline Maximum de-saturation & -6 & -4 & -6 & -5 \\
\hline Maximum pulse change & 34 & 26 & 32 & 35 \\
\hline \multicolumn{5}{|c|}{ Correlation-ship of the performance with $6 \mathrm{MWT}$ in terms of } \\
\hline a) cumulative change in pulse rate & & 0.92 & 0.96 & 0.93 \\
\hline b) cumulative change in $\mathrm{SpO}_{2}$ & & 0.88 & 0.95 & 0.89 \\
\hline
\end{tabular}

Showing that the 2CT with five movements matches best with 6MWT as regards the cumulative changes in $\mathrm{PR}_{\text {and }} \mathrm{SpO}$,

$\mathrm{PR}$, Pulse rate; $\mathrm{SpO}_{2}$, arterial oxygen saturation.

$(\mathrm{r}=0.96,0.95)$ with the same after 6MWT (table 2). Similarly, in another pilot observation on 20 volunteers, the duration taken for the exercise showed no effective correlation ( $\mathrm{r}=-0.287, \mathrm{r}=-0.284$, respectively for change in pulse rate and $\mathrm{SpO}_{2}$ ) with the cumulative change in $\mathrm{PR}$ and $\mathrm{SpO}_{2}$.

Two clinical assistants were trained to perform the test and allowed to do it independently on authorisation following satisfactory ratification of their performance as per the protocol on sham tests involving healthy volunteers and actual performance of the test on a few patients. The protocol decided was to do the tests serially as (1) two 2CTs for reproducibility and (2) a 2CT followed by 6MWT for comparison of the impact of 2CT to that of $6 \mathrm{MWT}$. There was no provision to repeat the $6 \mathrm{MWT}$ to account for any learning effect or possible, though unlikely, fatigue effect from performing the 2CT. Provision of stand-by oxygen and proper resuscitative measures were kept ready. They remained blindfolded about the details of ailments of the subjects selected.

\section{Selection of subjects}

Subjects were randomly selected by a physician on the basis of convenience. Any subject with the presence shortness of breath as the major symptom could be recruited. The exclusion criteria for the test included unwillingness to give a written consent, acute myocardial infarct in the preceding 4 weeks or perceived cardiovascular instability on clinico-radiological assessment or gross left ventricular dysfunction in echocardiography, gross musculoskeletal problem as advanced osteoarthritis of knees, vertigo or neurological disorder affecting normal movement, baseline hypoxaemia at rest $\left(\mathrm{SpO}_{2} \leq 90 \%\right)$, gross dyspnoea and fatigue from any reason, existing cardiac dysrhythmia and surviving on pacemaker beats, and any other systemic problems that can affect the exercise performance adversely.

\section{Patient and public involvement}

Subjects eligible for the study were directly informed regarding the need and rationality of the proposed exercise test by the consultant concerned before recruitment. The mode of performance and the risks involved were also elaborated before taking their consents. The result of the study was discussed with each of them soon after the performance by the same consultant.

\section{Looking for reproducibility and comparison with 6MWT}

Reproducibility of the test was verified on a set of patients by repeating the same test two times on the same day following a gap of at least 30 min or satisfactory stabilisation of $\mathrm{PR}$ and $\mathrm{SpO}_{2}$ whichever is later.

For comparison between 2CT and 6MWT, a different set of patients was selected. They underwent the 2CT first as per the protocol and then after subsequent stabilisation (having been debarred from any physical activities and no medication or beverage was allowed), 6MWT was done as per the standard protocol by the same set of clinical assistants in all of them. Following the performance of the 6MWT, the PR and the $\mathrm{SpO}_{2}$ were measured in protocol as is done after 2CT.

\section{Statistical analysis}

Statistical packages including, (GraphPad Prism, 7; Metboanalyst 4.0), were used for statistical analysis. Z-test, Student's t-test and Spearman's correlation were used to compare the two 2CT results as validation of reproducibility in the first set of volunteers. Further, post-exercise changes of $\mathrm{PR}$ and $\mathrm{SpO}_{2}$ following 2CT were compared with the same recorded after 6MWT. The comparisons were done in terms of 'moment to moment', 'mean maximum difference' (MMD) and 'mean cumulative difference' (MCD) of the changes. The MCD cumulative difference for each patient means the sum up of all the moment to moment differences for $\mathrm{PR}$ or $\mathrm{SpO}_{2}$. The 


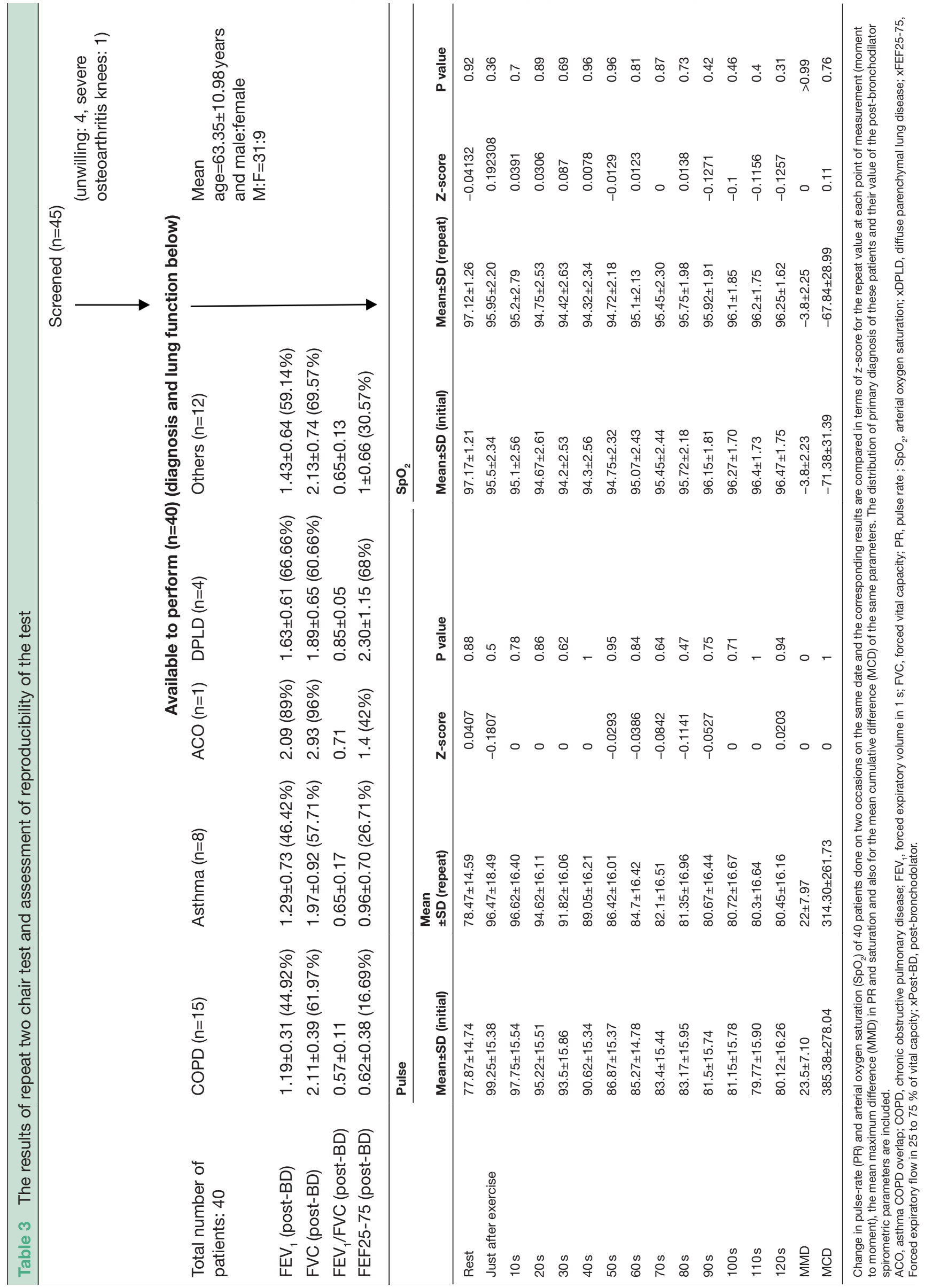



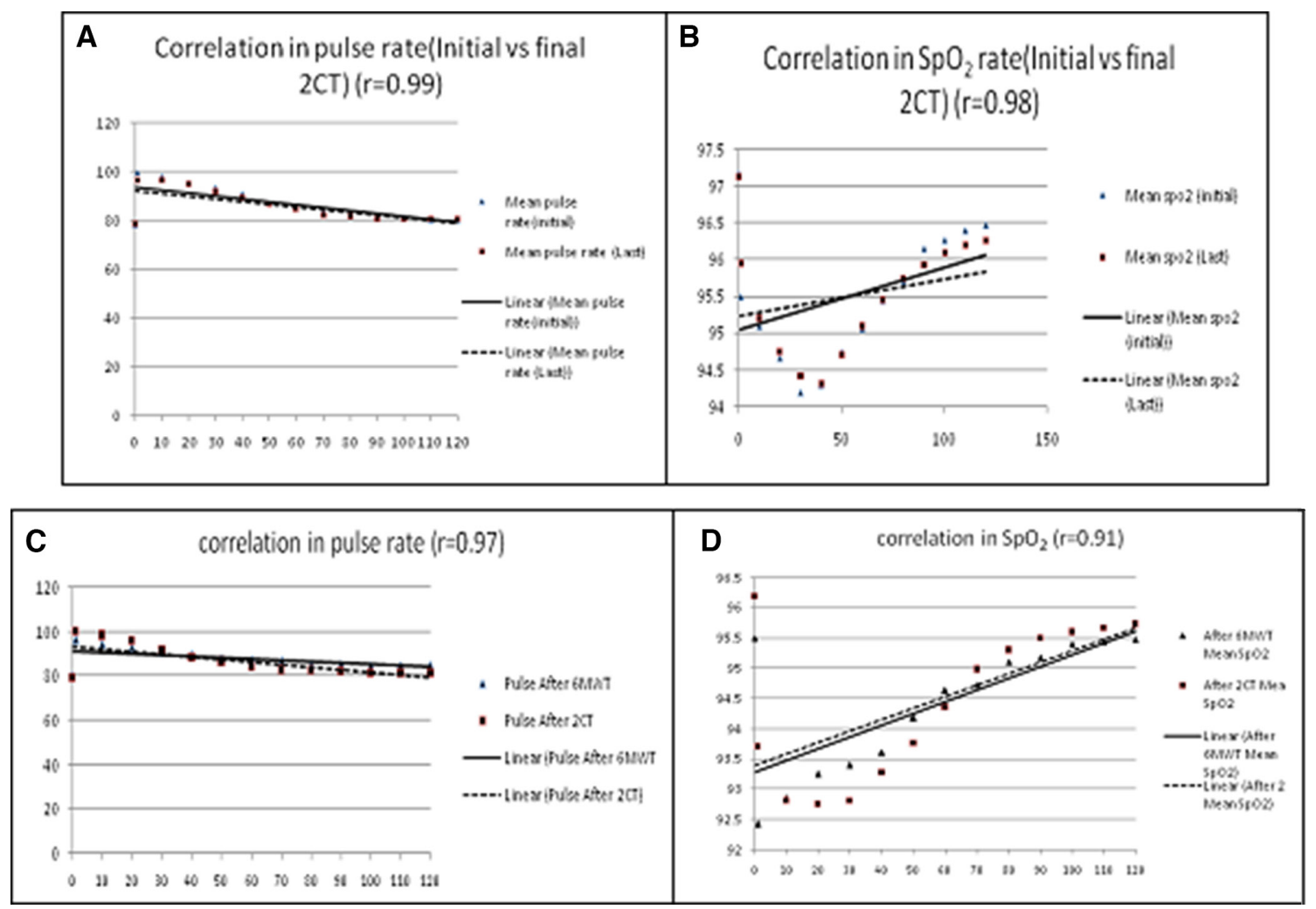

Figure 1 ( $A$ and $B$ ) Elaborates corelationship between the initial and the repeat tests as regards the changes in PR (A) and $\mathrm{SpO}_{2}$ (B) with the correlation coefficient $(r)$ being 0.99 and 0.98 respectively signifying excellent reproducibility. (C and $\left.D\right)$ Similar corelationships ( $r$ being 0.97 and 0.91 respectively) for post-exercise changes in pulse rate (PR) (C) and arterial oxygen saturation $\left(\mathrm{SpO}_{2}\right)$ (D) between $2 \mathrm{CT}$ and 6MWT suggest excellent comparability. (2CT, two chair test, PR, pulse-rate; $\mathrm{SpO}_{2}$, arterial oxygen saturation).

Spearman's correlation equation was also applied to see the relationship between the changes.

Principal component analysis (PCA), an unsupervised classification modelling method, aims to find the directions that best explain the variance in a data set (without referring to class labels) were also done. The data are summarised into much fewer variables which are weighted average of the original variables.

\section{RESULTS}

All the patients recruited for the study, completed five movements uneventfully. None of them needed supplemental oxygen or any medication. The results show reproducibility of the 2CT in all the 40 patients without any significant statistical difference between the initial and the repeat 2CT results (table 3). There was excellent correlations between the initial and repeat test in terms of $\mathrm{PR}(\mathrm{r}=0.99)$ and $\mathrm{SpO}_{2}(\mathrm{r}=0.98)$ (figure 1A,B). The PCA also shows complete overlapping of the samples from two independent tests (figure 2), confirming no significant difference between them.

The result of comparison between the post-exercise changes in the same parameters $\left(\mathrm{PR}\right.$ and $\left.\mathrm{SpO}_{2}\right)$ in another set of patients $(\mathrm{n}=60)$ reflect that $2 \mathrm{CT}$ and $6 \mathrm{MWT}$ are

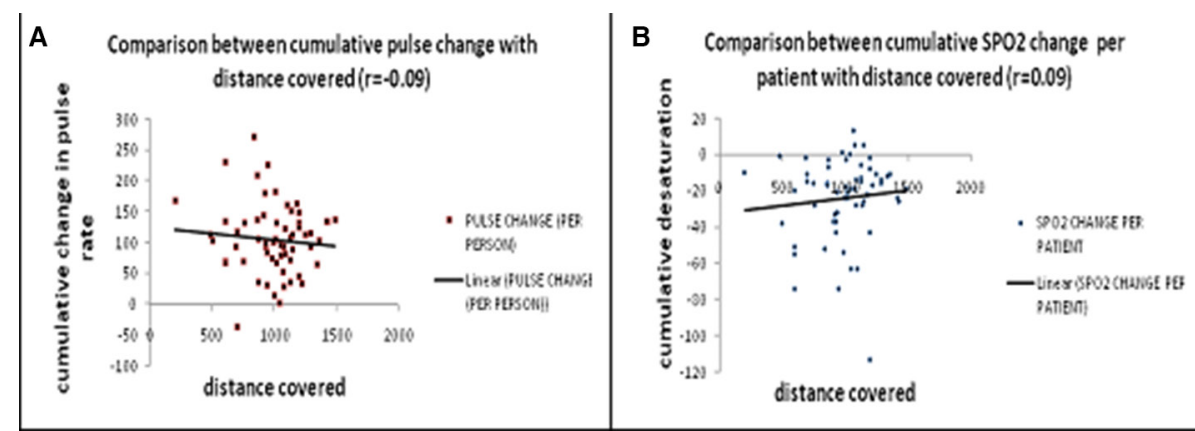

Figure 2 (2A and 2B) Elaborates the co-relationship of the post-exercise mean cumulative chnages in pulse rate and arterial oxygen saturation $\left(\mathrm{SpO}_{2}\right)$ with the distance covered in 6MWT (6 minute walk test) 


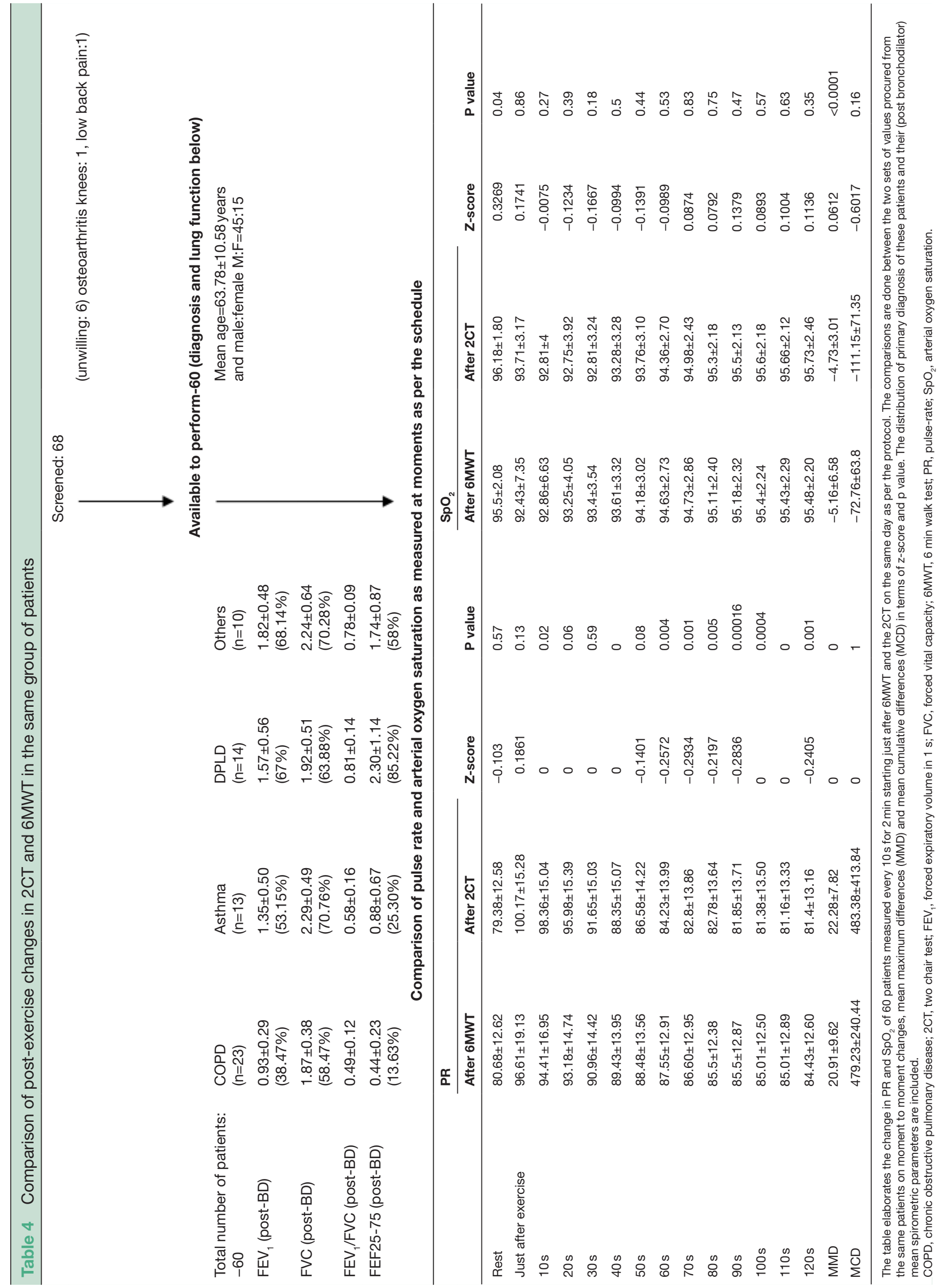




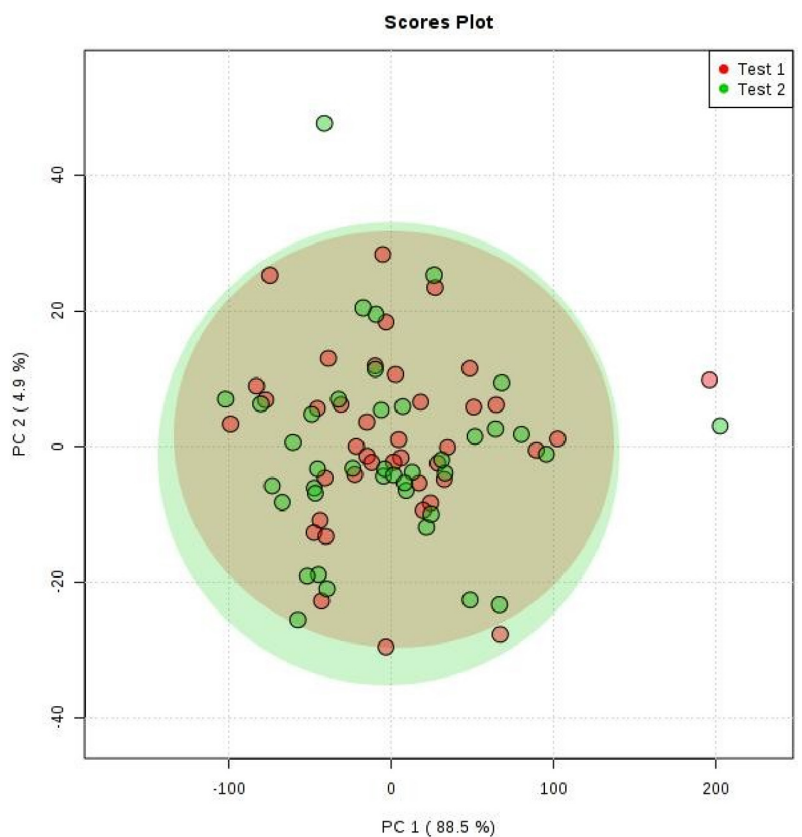

Figure 3 2CT versus 2CT: score scatter plots from the principal component analysis shows no difference of clustering between the initial (red) and repeat (green) two chair test performances by the same patients; the result support that the reproducibility is near perfect. The pulse rate and $\mathrm{SpO}_{2}$ values collected at every 10 seconds were used for the analysis. (2CT, two chair test.SpO ${ }_{2}$, arterial oxygen saturation) .

well comparable (table 4). The correlation between the changes observed after the 2CT and 6MWT were excellent $\left(\mathrm{r}=0.97\right.$ for $\mathrm{PR}$ and $\mathrm{r}=0.91$ for $\mathrm{SpO}_{2}$; figure $\left.1 \mathrm{C}, \mathrm{D}\right)$. The PCA also shows near complete overlapping of the samples from two independent tests (figure 3), confirming no significant difference between them.

The mean cumulative changes in $\mathrm{PR}$ and $\mathrm{SpO}_{2}$, both correlates poorly with the distance walked in 6MWT $(\mathrm{r}=0.09)$ and $(\mathrm{r}=0.09)$, respectively (figure 2A,B).

\section{DISCUSSION}

Our objective was to establish the reproducibility of 2CT and its comparability to 6MWT. The reproducibility has been established directly by four statistical end points as the $\mathrm{p}$ values $<0.05$, the $\mathrm{z}$-scores remained close to zero for all the comparisons, excellent corelationships between the initial repeat $\mathrm{PR}$ and $\mathrm{SpO}_{2}$ (figure 1A,B) and complete overlapping of the results in PCA (figure 2).

The comparison between 2CT and 6MWT was possible as regards their impact on the post-exercise $\mathrm{PR}$ and $\mathrm{SpO}_{2}$ on same patients $(n=60)$ that underwent both the exercises. The same statistical parameters of comparison as $\mathrm{p}$ value and z-score (table 4), r (figure 1C,D) and PCA overlapping (figure 3) are again suggestive of excellent similarity between the two sets of results. Presuming the mean change of any measurement after 6MWT as reference, the values of the same measurement in 2CT falling within $1 \mathrm{SD}$ of the former suggests an acceptable quantitative similarity. Thus, the observed z-scores close to 0 or smaller than $1 \mathrm{SD}$ have proved the excellent comparability in between 2CT and 6MWT as regards the measured post-exercise impact. Again, the excellent corelationship $(\mathrm{r}=0.97$ and $\mathrm{r}=0.91$, respectively) for $\mathrm{PR}$ and $\mathrm{SpO}_{2}$ (figure 1C,D) suggest similar trend of change for them. This comparability between the two tests again endorses the reproducibility of 2CT indirectly since 6MWT itself is found to be highly and even better reproducible than forced expiratory volume in $1 \mathrm{~s}^{22} 23$ The observation is unlikely to have been influenced by any change in the physiological status as both the tests were done on the same patients on the same day within 30 min of each other without any exercise, feeding or extra medication in between.

6MWT, a globally accepted and practiced test, was preceded by $12 \mathrm{~min}$ walk by Balke in early $60 \mathrm{~s}$ to evaluate functional capacity. ${ }^{24}{ }^{25}$ It was successful for assessing physical fitness of a healthy person and functional capacity of patients of chronic bronchitis. ${ }^{25} 26$ The 6MWT was introduced later as a better tolerated test; and a better marker of the capacity for daily activities. ${ }^{22} 27$

Being sub-maximal, both 2CT and 6MWT integrate global physiological response of all the systems involved in exercise (pulmonary, cardiac, circulatory, haematological, and neuromuscular, etc). However, while 6MWT marks the distance walked, the 2CT takes into account the post exercise changes of $\mathrm{PR}$ and $\mathrm{SpO}_{2}$ for $2 \mathrm{~min}$.

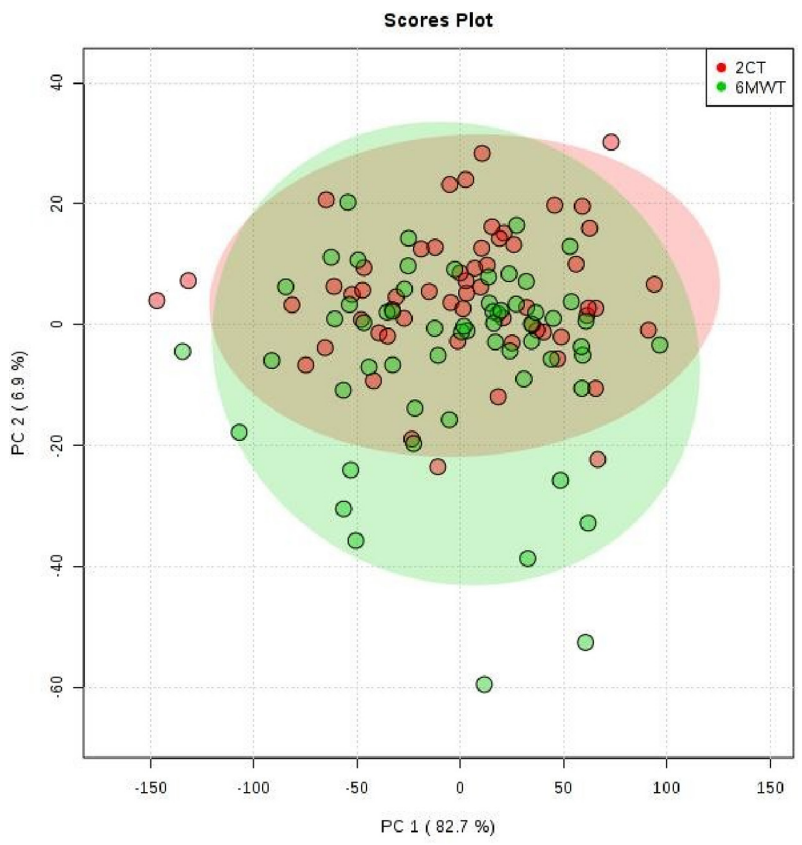

Figure 4 2CT versus 6MWT: score scatter plots in principal component analysis (PCA) show similar and overlapping clustering between the 2CT (red) and 6MWT (green) of the same patients supporting the fact that the two tests are highly comparable. Pulse rate and atrerial oxygen saturation $\left(\mathrm{SpO}_{2}\right)$ values from periodic intervals were used for the analysis as described in the protocol. 
The walking distance is likely contributed by some other factors than those that determine the changes in PR and the $\mathrm{SpO}_{2}$. This is apparent from the walking distance not correlating well to the MCD (mean cumulative change) of $\mathrm{PR}$ and $\mathrm{SpO}_{2}$ (figure 4A,B). Since $\mathrm{PR}$ and $\mathrm{SpO}_{2}$ relate to cardiopulmonary status both at rest and following exercise, their measurements in 2CT, therefore, reflect the recovery response of the cardiopulmonary stress been imposed by the exercise. Hence, the measurements made in 2CT are likely reflective of the equilibrium between the cardiopulmonary reserve and the uniformly imparted cardiopulmonary stress. The 2CT, therefore, needs to be ratified with standard physiological assessment parameters of cardiopulmonary exercise test. The inclusion of other variables as the dyspnoea status, quality of life, lung function and other invasive or non-invasive physiological measurements may turn useful.

The post-exercise change in PR and $\mathrm{SpO}_{2}$ are important. The exercise induced desaturation (measured just before and immediately following exercise) has been found related to poor lung function status, resting oxygen saturation and increased mortality in COPD. ${ }^{28}$ The heart rate recovery (difference between heart rate at peak exercise and following $1 \mathrm{~min}$ of termination of 6MWT) in patients with heart failure (irrespective of ejection fraction) has been found to be a more powerful prognostic indicator than the 6MWD as far as major cardiac events are concerned on longitudinal follow-up. ${ }^{29}$ Therefore, the post-exercise measurements of $\mathrm{PR}$ and $\mathrm{SpO}_{2}$ may be proved useful both in health and disease.

Some of our patients had delayed recovery beyond $2 \mathrm{~min}$. This phenomenon may have useful physiological significance and should be investigated adequately. A repeat test could reaffirm the revelation for both validation and comparison. Similarly, the protocol should have included a cross over attempt with 6MWT followed by 2CT to nullify the questionable influence of possible fatigue out of the first performance although we allowed adequate rest between the two and the baseline state before the tests were similar as regards the parameters noted.

The prospect of the test (2CT) needs attention and research. The assessment of deviation and the pattern of changes including the recovery response may be useful for diagnosis of poor cardiopulmonary reserve from a disease or its complications or presence of comorbidities. It can also reflect the impact of an intervention longitudinally. We have already used the concept of measuring the maximum deviation of $\mathrm{SpO}_{2}$ from baseline ('desaturation max') for treatment decision in patients of COPDpulmonary hypertension successfully. ${ }^{30}$ The test should be applied to normal population of different age groups in both the sexes to make a normal range to compare the abnormal results. Since the test requires a small space, it can be done at bedside and be used for real-time teleappreciation of patients' status. It is a simple, repeatable, reproducible and well-tolerated test demanding less logistic resource and cardiopulmonary reserve specific.
A repeat performance of the test may prove useful to follow a diseased. The test demands further validation in different disease states.

\section{Author affiliations}

${ }^{1}$ Pulmonology, Institute of Pulmocare and Research, DG-8, Action area 1, New Town, Kolkata, India

${ }^{2}$ Pulmonology, Institute of Pulmocare and Research, New Town, India

${ }^{3}$ Pulmonology, Institute of Pulmocare and Research, Kolkata, India

${ }^{4}$ Associate Prof, Cardiology, NRS Medical College, Kolkata, India

${ }^{5}$ Consultant Pulmonologist, Apollo Hospital, Kolkata, India

${ }^{6}$ Institute of Reproductive Medicine, Salt lake, Kolkata, Kolkata, India

${ }^{7}$ Pulmonologist, JNM Hospital, Kalyani, Nadia, India

${ }^{8}$ Consultant Cardiologists, Peerless Hospital, Kolkata, India

${ }^{9}$ Consultant Cardiologist, Fortes Hospital Anandapur, Kolkata, West Bengal, India

Contributors All authors (PB, DS, MP, DG, BM, SushmitaRC, SouravRC, PA, IH, DGR, SR) contributed to planning, conduct, reporting, conception and design, analysis and interpretation of data for this manuscript.

Funding The authors have not declared a specific grant for this research from any funding agency in the public, commercial or not-for-profit sectors.

Competing interests None declared.

Patient consent for publication Not required.

Ethics approval The study was approved by the Institutional Ethics Committee (DCGI approved IRB (IEC) ECR/159/Inst/WB/2013/RR-16 issued under Rule 122DD of the Drugs and Cosmetics Rules 1945 dated 21 February 2017). The recruitment was done from outpatient department of the institute following procurement of a proper written consent.

Provenance and peer review Not commissioned; externally peer reviewed.

Data availability statement All data relevant to the study are included in the article or uploaded as supplementary information.

Open access This is an open access article distributed in accordance with the Creative Commons Attribution Non Commercial (CC BY-NC 4.0) license, which permits others to distribute, remix, adapt, build upon this work non-commercially, and license their derivative works on different terms, provided the original work is properly cited, appropriate credit is given, any changes made indicated, and the use is non-commercial. See: http://creativecommons.org/licenses/by-nc/4.0/.

\section{ORCID iD}

Parthasarathi Bhattacharyya http://orcid.org/0000-0001-7246-5315

\section{REFERENCES}

1 Sinclair DJ, Ingram CG. Controlled trial of supervised exercise training in chronic bronchitis. Br Med J 1980;280:519-21.

2 Roomi J, Johnson MM, Waters K, et al. Respiratory rehabilitation, exercise capacity and quality of life in chronic airways disease in old age. Age Ageing 1996;25:12-16.

3 O'Keeffe ST, Lye M, Donnellan C, et al. Reproducibility and responsiveness of quality of life assessment and six minute walk test in elderly heart failure patients. Heart 1998;80:377-82.

4 Spence DP, Hay JG, Carter J, et al. Oxygen desaturation and breathlessness during corridor walking in chronic obstructive pulmonary disease: effect of oxitropium bromide. Thorax 1993;48:1145-50.

5 Solway S, Brooks D, Lacasse Y, et al. A qualitative systematic overview of the measurement properties of functional walk tests used in the cardiorespiratory domain. Chest 2001;119:256-70.

6 Kadikar A, Maurer J, Kesten S. The six-minute walk test: a guide to assessment for lung transplantation. J Heart Lung Transplant 1997;16:313-9.

7 Holden DA, Rice TW, Stelmach K, et al. Exercise testing, 6-min walk, and stair climb in the evaluation of patients at high risk for pulmonary resection. Chest 1992;102:1774-9.

8 Criner GJ, Cordova FC, Furukawa S, et al. Prospective randomized trial comparing bilateral lung volume reduction surgery to pulmonary rehabilitation in severe chronic obstructive pulmonary disease. $\mathrm{Am} J$ Respir Crit Care Med 1999;160:2018-27.

9 Hajiro T, Nishimura K, Tsukino M, et al. Analysis of clinical methods used to evaluate dyspnea in patients with chronic obstructive pulmonary disease. Am J Respir Crit Care Med 1998;158:1185-9. 
10 Gulmans VA, van Veldhoven $\mathrm{NH}$, de Meer $\mathrm{K}$, et al. The six-minute walking test in children with cystic fibrosis: reliability and validity. Pediatr Pulmonol 1996;22:85-9.

11 Peeters $P$, Mets T. The 6-minute walk as an appropriate exercise test in elderly patients with chronic heart failure. $J$ Gerontol A Biol Sci Med Sci 1996;51:M147-51.

12 Montgomery PS, Gardner AW. The clinical utility of a six-minute walk test in peripheral arterial occlusive disease patients. J Am Geriatr Soc 1998;46:706-11.

13 Cahalin LP, Mathier MA, Semigran MJ, et al. The six-minute walk test predicts peak oxygen uptake and survival in patients with advanced heart failure. Chest 1996;110:325-32.

14 Cote CG, Celli BR. In patients with COPD, the 6 minute walking distance is a better predictor of health care utilization than FEV1, blood gases, and dyspnea. Eur Respir J 1998;383.

15 Kessler R, Faller M, Fourgaut G, et al. Predictive factors of hospitalization for acute exacerbation in a series of 64 patients with chronic obstructive pulmonary disease. Am J Respir Crit Care Med 1999;159:158-64.

16 Cahalin L, Pappagianopoulos P, Prevost S, et al. The relationship of the 6-min walk test to maximal oxygen consumption in transplant candidates with end-stage lung disease. Chest 1995;108:452-9.

17 Butland RJ, Pang J, Gross ER, et al. Two-, six-, and 12-minute walking tests in respiratory disease. Br Med J 1982;284:1607-8.

18 Niederman MS, Clemente PH, Fein AM, et al. Benefits of a multidisciplinary pulmonary rehabilitation program. improvements are independent of lung function. Chest 1991;99:798-804.

19 Guyatt GH, Pugsley SO, Sullivan MJ, et al. Effect of encouragement on walking test performance. Thorax 1984;39:818-22.

20 Ats Committee on proficiency standards for clinical pulmonary function laboratories. ats statement: guidelines for the six-minute walk test. Am J Respir Crit Care Med 2002;166:111-7.
21 Léger LA, Lambert J. A maximal multistage 20-m shuttle run test to predict VO2 max. Eur J Appl Physiol Occup Physiol 1982;49:1-12.

22 Singh SJ, Morgan MD, Scott S, et al. Development of a shuttle walking test of disability in patients with chronic airways obstruction. Thorax 1992;47:1019-24.

23 Puhan MA, Siebeling L, Zoller M, et al. Simple functional performance tests and mortality in COPD. European Respiratory Journal 2013;42:erj01316-2012:956-63.

24 Balke B. A simple field test for the assessment of physical fitness. Civil Aerospace Medical Institute, 1963.

25 Cooper $\mathrm{KH}$. A means of assessing maximal oxygen intake. Correlation between field and treadmill testing. JAMA 1968;203:201-4.

26 McGavin CR, Gupta SP, McHardy GJ. Twelve-minute walking test for assessing disability in chronic bronchitis. $\mathrm{Br}$ Med $J$ 1976;1:822-3.

27 Solway S, Brooks D, Lacasse Y, et al. A qualitative systematic overview of the measurement properties of functional walk tests used in the cardiorespiratory domain. Chest 2001;119:256-70.

28 Dogra AC, Gupta U, Sarkar M, et al. Exercise-Induced desaturation in patients with chronic obstructive pulmonary disease on six-minute walk test. Lung India 2015;32:320.

29 Cahalin LP, Arena R, Labate V, et al. Heart rate recovery after the 6 min walk test rather than distance ambulated is a powerful prognostic indicator in heart failure with reduced and preserved ejection fraction: a comparison with cardiopulmonary exercise testing. Eur J Heart Fail 2013;15:519-27.

30 Bhattacharyya P, Sengupta S, Bhattacharjee PD, et al. Post exercise desaturation can help identifying treatment responders of COPD pulmonary hypertension in real world: an appraisal. J Pulm Respir Med 2018;8:469 